\title{
Retrospective and prospective short-term memory in delayed response tasks in rats
}

\author{
J. S. COHEN, R. GALGAN, and D. FUERST \\ University of Windsor, Windsor, Ontario, Canada
}

\begin{abstract}
Separate groups of rats were trained and tested on asymmetrically and symmetrically reinforced successive delayed matching-to-sample (DMTS) or delayed discrimination (DD) tasks in Experiment 1. Each rat received training and testing on symmetrically reinforced DMTS and DD tasks in Experiment 2. The only difference between each task was that the rats had to respond correctly to a light or tone test stimulus, $S_{2}$, if it matched a light or tone sample stimulus, $S_{1}$, in DMTS, but could respond to either $S_{2}$ if $S_{1}$ had been a particular stimulus in DD. Only correct leverpresses were reinforced in the asymmetrically reinforced version of each task. Both correct presses and correct omissions were reinforced in the symmetrically reinforced version of each task. Response biases to leverpress during tests for delayed responding to $S_{1}$ were reduced in both symmetrically reinforced tasks, but only in the DD task did such contingencies produce consistently poorer performance in responding to either $S_{1}$ in Experiment 1. Declines in accuracy of performance that occurred in both experiments were greater to the visual than to the auditory $S_{1}$ only in the DMTS tasks with increased intervals between $S_{1}$ and $S_{2}$. A third experiment, in which rats had to respond to $S_{2}$ if it matched $S_{1}$ (DMTS) or if $S_{2}$ mismatched $S_{1}$ (DMmTS), was carried out. Modality of $S_{1}$ similarly affected accuracy of delayed responding in each task, as in the first two experiments. Methodological and theoretical implications of these results are discussed in terms of Honig and Thompson's (1982) dual-process theory of working memory.
\end{abstract}

Honig and Thompson (1982) distinguish between retrospection, memory of a previous stimulus, and prospection, retention of an instruction for future responding to the previous stimulus, in short-term memory of nonhuman animals. The form of a delayed response task may promote one type of processing over the other. In delayed conditional discrimination (DCD), in which responding to a stimulus from a set of stimuli (Blough, 1959) or to a singly presented stimulus (Konorski, 1959) is based on actual or symbolic matching to a prior sample stimulus, retrospection may be necessary. Prospection may occur when either the initial stimulus provides all the information for responding, as in delayed simple discrimination (DD), or after the organism has determined if the test stimulus matches the sample in DCD. Furthermore, retrospection may be more difficult than prospection if the latter requires less information to be retained. Increasing retention intervals between sample and test stimuli does reduce performance more drastically in delayed con-

\footnotetext{
Support for this research was obtained from the National Science and Engineering Council of Canada in the form of an operating grant to the first author (No. A8249) and a graduate scholarship to the second author (No. 800). Experiment 1 served as a thesis in partial fulfillment for the MA degree given to the second author by the University of Windsor. Experiment 1 was initially presented at the Psychonomic Society meetings in San Antonio, Texas, November 1984. Experiments 2 and 3 were presented at the Canadian Psychological Association meetings in Halifax, June 1985. The authors wish to thank W. Honig and R. Weisman for their thoughtful criticisms of this study. Reprint requests should be addressed to J. S. Cohen, Department of Psychology, University of Windsor, Windsor, Ont., Canada N9B 3P4.
}

ditional than in delayed simple discrimination, in pigeons (Honig \& Wasserman, 1981; Smith, 1967). Increasing retention intervals after the sample stimuli also disrupts matching more than does increasing them after the conditional stimulus, in pigeons (Honig \& Dodd, 1983; Weisman \& DiFranco, 1981).

Differences in general performance between DCD and DD or conditional delayed discriminations may provide only weak evidence for the dual-process theory, however. Delayed matching is not always as easily disrupted by increased retention intervals as was the case in the studies already cited. Well-trained pigeons (Grant, 1976) or those presented with sample stimuli of longer durations (16 sec) (Shimp \& Moffitt, 1977, Experiment 1) maintain highly accurate matching. Furthermore, prospection could conceivably produce as poor, or worse, retention than retrospection, if the instructions to be rehearsed are as, or more, complex than the stimuli to be remembered. Consequently, the effects of the physical characteristics of the sample stimulus and the nature of the response rules in DCD and DD tasks may better differentiate one process from the other. If retrospection predominantly occurs in DCD, the nature of the sample stimulus, rather than the response rules signaled by it, should affect delayed performance. If prospection occurs mainly in DD tasks, however, the nature of the response rules, rather than the characteristics of the sample stimulus, should affect delayed performance.

In this study, we used the fact that rats match better to a tone than to a light sample stimulus in delayed matching-to-sample (DMTS) tasks (Cohen, Escott, \& Ric- 
ciardi, 1984; Wallace, Steinert, Scobie, \& Spear, 1980), to test the above hypothesis. In those previous studies, rats had to respond to a second light or tone test stimulus, $S_{2}$, for reinforcement if it matched a first light or tone sample stimulus, $S_{1}$. Accuracy for delayed matching to $S_{1}$ was determined by increasing the retention interval (RI) between $S_{1}$ and $S_{2}$. In the present study, we presented the same sequential pairs of light and tone stimuli but varied the contingencies for reinforcement so that, in DMTS, responding on $S_{2}$ was reinforced if it matched $S_{1}$, but in $D D$, responding on $S_{2}$ was reinforced only if it followed a specific $S_{1}$. We predicted that modality of $S_{1}$ would affect performance only in DMTS. In the first experiment, we also varied the complexity of the response rule for reinforcement. The simpler, and presumably easier to prospect, rule merely required the animal to leverpress on $S_{2}$ after an appropriate $S_{1}$. Since responding on only half the stimulus pairs would be reinforced, this rule was for asymmetrical reinforcement. The more complex rule allowed animals to obtain reinforcement for both correctly responding to $S_{2}$ and correctly omitting a response to $S_{2}$. Since animals could obtain reinforcement on each stimulus pair, this rule was for symmetrical reinforcement. We expected that increased complexity of the latter instructional code would inversely affect delayed performance more in DD than in DMTS. Cohen et al. (1984) found that symmetrical reinforcement reduced a bias to leverpress with increased RIs in DMTS, which enhanced already better delayed matching to the tone $S_{1}$.

The experiments in the present study were also designed to permit analysis of performance by measures of signal sensitivity derived from the theory of signal detection (TSD). Although detailed accounts of TSD exist (e.g., McNicol, 1972), we present a brief description of and rationale for this mode.

TSD was originally developed to assess human capacity for signal detection independent of response bias (Swets, Tanner, \& Birdsall, 1961; Tanner \& Swets, 1954). Unlike methods from classical psychophysics, which attempt to eliminate response bias, TSD procedures encourage the subject to adopt a wide range of biases or criteria. Manipulation of the payoff structure for making correct and incorrect responses and varying the probability of a signal occurring on a trial are two common methods used to promote changes in criteria. Criteria range from the more conservative, where subjects try to reduce incorrect positive responses (false alarms) at the expense of obtaining correct positive responses (hits), to the more lax, where subjects try to obtain hits, even though false alarms will increase. Proportions of hits and false alarms at each criterion level are measured to form a curvilinear function, the receiver operating curve (ROC), from these data points. ROC defines a unitary signal-sensitivity measure as the area underlying this curve. The proportion of the area, $P(A)$, beneath ROC ranges from .50 , no signal detection, to 1.00 , perfect signal detection. $P(A)$ rather than $d^{\prime}$, the standard score form, is preferred when assumptions of normality and homogeneity of variance of sensory events of signal and noise in the subject are in doubt (McNicol, 1972).

A unitary signal-sensitivity measure from a range of criteria avoids overestimation of sensitivity from conservative criteria and underestimation of sensitivity from lax criteria. According to D'Amato (1970), analyzing $S_{1}$ retention by TSD methods is a logical application of this theory. Such methods should especially be used in tasks in which response biases may be prevalent. Weiskrantz (1968) suggests that Konorski's variant of DCD is more likely to produce a bias for positive responding than is Blough's procedure, because reinforcement is asymmetrically available in the former. Even when reinforcement is symmetrically available in the Konorski DCD, such biases may be reduced but not eliminated in rats (Cohen et al., 1984). We decided to use Konorski's delayed matching task, despite its inherent characteristic to produce response biases, because rats acquire it more easily than they do Blough's version (Wallace et al., 1980). To control for such biases, we employed a TSD sensitivity measure. Since promoting various criterion levels in rats is, at best, difficult, we used an estimation of $P(A)$, $P(\bar{A})$, from only one set of hits and false alarms at each RI. Calculation of $P(\bar{A})$ is described in the first experiment.

\section{EXPERIMENT 1}

\section{Method}

\section{Design}

Two separate groups of rats received identical pairs of sequential stimuli, but one group acquired a successive delayed matchingto-sample task (DMTS) and the other, a delayed simple discrimination (DD). Each rat acquired both the asymmetrically and symmetrically reinforced form of its particular task. A trial began with either onset of chamber lights or a tone $\left(S_{1}\right)$ followed by a 1-sec interval and then either of the same two stimuli $\left(S_{2}\right)$ accompanied by a retractable lever. Thus, there were four different pairs of stimuli: light-light ( $\mathrm{L}-\mathrm{L})$, tone-tone $(\mathrm{T}-\mathrm{T})$, light-tone $(\mathrm{L}-\mathrm{T})$, and tonelight (T-L). For reinforcement, animals in the DMTS task had to press to $S_{2}$ when it matched $S_{1}$ (L-L, T-T). No reinforcement was available on mismatching pairs ( $L-T, T-L)$ in the asymmetrically reinforced version of this task. In the symmetrically reinforced form of DMTS, reinforcement was also available if pressing was omitted to $S_{2}$ when it differed from $S_{1}$ (L-T, T-L). For reinforcement, animals in the DD group had to press to any $S_{2}$ if it followed a particular $S_{1}$. Pressing to $S_{2}$ after the other $S_{1}$ was not reinforced. In the symmetrically reinforced version, reinforcement was also contingent for not pressing to $S_{2}$ after the other $S_{1}$. Half the animals in the DD group first learned, and were tested on, the asymmetrical and symmetrical versions of DD in which pressing to $S_{2}$ following a light $S_{1}$ was reinforced. For the other DD animals, tone $S_{1}$ signaled reinforcement for a leverpress. After learning and being tested on this task, the animals were trained and tested on the two forms of the reversed DD task in which pressing to the other $S_{1}$ was reinforced.

A multiple baseline procedure was used during the delay test phase following acquisition of each form of DMTS and DD task. Blocks of test trials, each containing fixed increased RIs of 5, 10, and $20 \mathrm{sec}$, were separated by reacquisition blocks containing 1-sec RIs. Data from each block of test trials were compared with baseline data from the previous block of reacquisition trials. These procedures were designed to overcome any differences in acquisition be- 
tween various forms of the DMTS or DD tasks and to allow us to analyze matching to, or differentiation of $S_{1}$ by a signal sensitivity score, $P(\bar{A})$, as described by $\mathrm{McNicol}(1972)$. We predicted that increased RIs would reduce $P(\bar{A})$ scores more to light than to tone $S_{1}$ only in the DMTS group, but would reduce $P(\bar{A})$ scores more for symmetrical than for asymmetrical reinforcement primarily in the DD task.

\section{Subjects}

Twelve male albino (Wistar) rats, approximately $90-150$ days old at the start of the experiment, served as subjects. They came from our breeding colony and were individually housed with food continuously available in their home cages. They were maintained on a water-deprivation schedule in which water was available for 15-20 min approximately $30 \mathrm{~min}$ after each daily session. Animals were divided into equal DMTS and DD groups. Two DMTS animals failed to acquire the task within 60 sessions and were replaced by two animals that had served as subjects in an operant discrimination task with both stimuli as components of a compound $\mathbf{S}+$. These replacement rats were considered acceptable because they had experienced the same prediscrimination procedures to learn to press a retractable lever within $5 \mathrm{sec}$ every $30 \mathrm{sec}$, but had not received any retention intervals during compound discrimination training.

\section{Apparatus}

The apparatus was that described by Cohen et al. (1984). It consisted of four commercially available (BRS/LVE) operant chambers, each containing a retractable lever and a $.05-\mathrm{ml}$ water dipper on one of the walls and five lights and a tone generator in the ceiling. Each operant chamber was enclosed in a BRS/LVE sound- and light-attenuating isolation chamber, whose ventilation fan produced constant 55-dB masking noise. An external speaker in the running room emitted $62-\mathrm{dB}$ white noise to further mask sounds from programming equipment in the adjacent room. Two 2.5-sec stimulus events, onset of ceiling lights and a 75- $\mathrm{dB} 2.8-\mathrm{kHz}$ tone, were used. Operant chambers remained dark during intertrial and retention intervals. Reinforcement was $.05 \mathrm{ml}$ of sweetened water $(.10 \%$ sodium cyclamate, w/v) delivered by the dipper.

\section{Procedure}

The animals were randomly divided into two separate task groups of six animals each. Each animal received initial approximation training to press a lever every $30 \mathrm{sec}$ within $5 \mathrm{sec}$ of its presentation in the chamber. This training was followed by 4 weeks of preliminary discrimination training, in which positive and negative stimulus pairs were presented in alternating blocks of 10 or 5 trials per session (100 trials) in either the DMTS or DD task. These procedures were designed specifically to reduce the number of animals that might fail to acquire the task. Since these procedures are described elsewhere (Cohen et al., 1984), the following description concentrates on the final training and testing phases of DMTS and DD.

Discrimination training. Animals in both the DMTS and DD groups received sequential pairs of 2.5 -sec light and tone stimuli in four possible orders: $\mathrm{L}-\mathrm{L}, \mathrm{T}-\mathrm{T}, \mathrm{L}-\mathrm{T}$, and T-L. The two stimuli were separated by a 1 -sec retention interval (RI) during training. Onset of $S_{2}$ was accompanied by a lever that remained out up to $5 \mathrm{sec}$. Initiation of each trial occurred with onset of $S_{1}$ every $24 \mathrm{sec}$ after termination of $S_{2}$ of the preceding trial. Each session contained 100 trials. The four different sequential pairs of stimuli appeared equally in each session in a semirandom order, so that matching or mismatching pairs, or the same $S_{1}$ of either kind of pair, did not occur more than three times in a row.

Half the animals in each task group were first trained and tested on the asymmetrically reinforced version of their respective task and then trained and tested on the symmetrically reinforced form. The remaining animals were trained and tested first on the symmetrically and then on the asymmetrically reinforced version of their tasks. DMTS animals received these two stages of training and test- ing, but $\mathrm{DD}$ animals received an extra training and testing phase. In the first phase of training, half the DD animals were trained with the light $S_{1}$ as positive for leverpressing and half were trained with tone $S_{1}$ as positive. After receiving training and testing under each type of reinforcement symmetry, DD animals were trained and tested on a reversal of their original task. If pressing to $S_{2}$ following a light (or tone) $S_{1}$ had been reinforced in the first discrimination, now pressing to $S_{2}$ following a tone (or light) $S_{1}$ was reinforced. Of the three DD rats trained to press after light $S_{1}$, two were first symmetrically reinforced and then asymmetrically reinforced; the third rat had the order reversed. Of the DD rats that first acquired the tone $S_{1}$ discrimination, two received asymmetrical and then symmetrical reinforcement. To minimize the disruptive effects of changing from one $S_{1}$ discrimination to another in the DD task, we tried to keep the same type of reinforcement contingency in the initial version of the new $S_{1}$ discrimination that had existed in the second stage in the first $S_{1}$ discrimination. This ABBA sequence for changing reinforcement symmetry was carried out for all rats switched from light $S_{1}$ to tone $S_{1}$ discrimination. Two rats, initially trained with the tone $S_{1}$ discrimination for symmetrical reinforcement on the second stage, however, failed to acquire the reversed light $S_{1}$ discrimination for symmetrical reinforcement in the first stage of the second training phase within seven sessions. Therefore, we retrained and tested these animals on the asymmetrically reinforced form of the light $S_{1}$ discrimination first.

Every rat in both task groups received a 3-day rest period in its home cage with free access to food and water before each change in training and testing conditions.

In the asymmetrically reinforced form of DMTS, pressing to $\mathbf{S}_{\mathbf{2}}$ produced reinforcement when it matched $S_{1}$ (L-L, T-T), but no reinforcement could be obtained when $S_{2}$ differed from $S_{1}$ (L-T, T-L). In the symmetrically reinforced version of DMTS, not pressing to $S_{2}$ when it differed from $S_{1}$ (L-T, T-L) would produce reinforcement, as did pressing when $S_{2}$ matched $S_{1}(L-L, T-T)$. In the asymmetrically reinforced version of the visual $S_{1} D D$, pressing to either $S_{2}$ following a light $S_{1}(L-L, L-T)$ was reinforced. No reinforcement occurred for pressing on pairs initiated by tone $S_{1}$ (T-T, T-L). In the symmetrically reinforced version, omitting a press on pairs initiated by the tone $S_{1}$ was also reinforced. In the auditory $S_{1} D D$, pressing to either $S_{2}$ following a tone $S_{1}$ (T-T, T-L) rather than a light $S_{1}$ (L-L, L-T) was reinforced. Omitting a press to either $S_{2}$ following light $S_{1}$ was also reinforced in the symmetrically reinforced version of this task. In either DMTS or DD tasks, animals could be reinforced only on half the trials in a session for asymmetrical reinforcement but on every trial for symmetrical reinforcement.

An animal was considered to have acquired its specific discrimination task when it pressed correctly on $75 \%$ of its total presses in each of two successive sessions. To reach this criterion, the animal had to make at least 30 reinforced and 10 nonreinforced presses per session. This criterion insured that animals did not reach a high proportion of correct presses when they failed to respond correctly for as many or more times.

$S_{1}$ delay tests. Delayed responding to $S_{1}$ was assessed at RIs of 5,10 , and $20 \mathrm{sec}$ on each task in the following manner. After reaching criterion on a particular version of the discrimination task, each rat received the first block of retention test trials for two sessions ( 200 trials) with a 5 -sec RI in each trial, followed by a series of reacquisition sessions to criterion with 1 -sec RIs. A second block of delay test trials for two sessions, with a 10-sec RI in each trial, was presented and followed by a second series of reacquisition sessions to criterion. A final block of delay test trials for two sessions, with a $20-\mathrm{sec}$ RI in each trial, followed the previous reacquisition sessions. If the animal was to be either switched to another form of the same discrimination or, in the case of DD animals, retrained on the reversed discrimination, a final series of reacquisition sessions was run.

Data analysis. Each block of training trials at criterion (200 trials) provided baseline performance for comparison with the following block of 200 delay test trials. Each delay test and baseline block of trials contained 100 matching and 100 nonmatching stimulus 
pairs, half of each beginning with the auditory $S_{1}$ and half beginning with the visual $S_{1}$. For each DMTS animal, proportions of reinforced and nonreinforced leverpresses on each type of stimulus pair for each $S_{1}$ were recorded. In each baseline and test block, DMTS animals could make 50 reinforced presses each to L-L and $\mathrm{T}$ - $\mathrm{T}$ pairs and 50 nonreinforced presses each to $\mathrm{L}-\mathrm{T}$ and $\mathrm{T}$ - $\mathrm{L}$ pairs. For DD animals, number of reinforced and nonreinforced responses was determined by the nature of $S_{1}$. Therefore, proportions of reinforced and nonreinforced presses out of 100 trials to each $S_{1}$ were noted. For DD animals trained on the visual $S_{1} D D, 100$ reinforced presses to stimulus pairs containing a light $S_{1}(\mathrm{~L}-\mathrm{L}$ and $\mathrm{L}-\mathrm{T}$ ) and 100 nonreinforced presses to tone $S_{1}$ pairs (T-T and T-L) could be made on each block. For DD animals trained on the auditory $S_{1}$ discrimination, 100 reinforced presses to tone $S_{1}$ pairs (T-T, T-L) and 100 nonreinforced presses to light $S_{1}$ pairs (L-L, L-T) could be made on each block.

Matching to, or differentiation of, $S_{1}$ in DMTS or DD, respectively, was determined by an estimate of stimulus sensitivity, $P(\bar{A})$, derived from signal detection theory (McNicol, 1972). $P(\bar{A})$ is an estimate of $P(A)$, the proportion of area lying under a receiver operating curve (ROC) determined by several pairs of proportions of correct or reinforced (hits) and incorrect or nonreinforced (false alarms) presses. Since $P(A)$ makes no assumptions about the distribution of sensory events of signal and noise in the subject, it can be determined graphically. $P(A)$ will range from .50 , no differentiation of, or matching to, $S_{1}$, to 1.00 , perfect differentiation of, or matching to, $\mathrm{S}_{1} . P(\bar{A})$ is determined from only one pair of proportions of hits and false alarms. Two possible linear ROC functions may be obtained from a single pair of hit and false-alarm proportions: one through that data point to a common 0 point and the other through it to a common point of unity. $P(\bar{A})$ is the average of the sum of areas lying under both lines which may be calculated algebraically as:

$$
P(\bar{A})=0.5\left[\left(4 x y-3 y-y^{2}-x^{2}+x\right) / 2 y(x-1)\right],
$$

where $y=$ proportion of hits and $x=$ proportion of false alarms.

Previous studies in DCD either were not appropriately designed to generate reliable proportions of hits or false alarms or, if so. used proportion of correct responses $[P(c)]$ or discrimination ratios (DR) of number of false alarms to number of hits instead of $P(\bar{A})$. By using blocks of 200 baseline and delay test trials, however, we could generate adequate proportions of hits and false alarms for calculating $P(\bar{A}) \mathrm{s} . P(\bar{A})$ is superior to $P(c)$ or $\mathrm{DR}$ as an index of delayed $S_{1}$ differentiation (DD) or matching (DMTS) because it takes into account both declines in hits as well as increases in false alarms. $P(c)$ and DR take into account only number of nonreinforced presses or false alarms. $P(c)$ or DR may be acceptable if the only source of change in stimulus differentiation or matching is due to changes in false alarms, as in two-alternative forced choice tasks (McNicol, 1972), such as simultaneous DMTS. To illustrate this point, consider a rat, trained in the successive DMTS, which adopts a strict criterion by obtaining only $40 \%$ hits and no false alarms. Both $P(c)$ and DR overestimate its performance with perfect scores of 1.00 and 0 , respectively, but $P(\bar{A})$ shows a slightly poorer performance of .84 . Now, if the rat uses a lax criterion by obtaining $99 \%$ hits and $60 \%$ false alarms, $P(\bar{A})$ is still .84 but $P(c)$ and DR underestimate performance with scores of .62 and .61 , respectively. By using a combined proportion of correct responses and omissions, $P(C)$, we will not obtain as great variations with changes in criteria, but, in our example, $P(C)$ yields lower scores of .70 and .74 than does $P(\bar{A})$. Statistical comparisons among these measures (Cohen \& Fuerst, 1985) for data from Cohen et al. (1984) support these differences.

\section{Results}

Proportion of reinforced (hits) and nonreinforced (false alarms) presses, from which $P(\bar{A})$ scores were derived, was calculated for each animal at each baseline and test block. These three proportion measures were further changed into arcsin transformations to normalize the data and reduce its heterogeneity of variance for parametric statistical analysis, as suggested by Winer (1971). The data were graphed in raw score form, however, for ease of explication. A five-way ANOVA [task (DMTS, DD) $\times$ reinforcement symmetry (asymmetrical, symmetrical) $\times S_{1}$ modality (light, tone) $\times$ blocks (baseline, test) $\times \mathrm{RI}(5$, $10,20 \mathrm{sec})]$ with repeated measures on the last four factors was run on each transformed dependent variable. We followed Ferguson's (1981) suggestion of using NewmanKeuls tests rather than more powerful orthogonal comparisons for individual comparisons.

\section{Proportion of Hits (Reinforced Leverpresses)}

Figure 1 shows the proportion of hits and false alarms (nonreinforced presses) at each baseline (1-sec RI) and test block (5-, 10-, 20-sec RI). As seen in Figure 1, reinforced responses to the light $S_{1}$ in the DMTS task (on L-L pairs) and DD task (both L-L and L-T pairs) declined on test blocks from baseline levels only for symmetrical reinforcement. No other consistent changes in hits to either the light $S_{1}$ in asymmetrically reinforced tasks or the tone $S_{1}$ in either form of each task were observed. A significant interaction of $S_{1}$ modality $\times$ reinforcement symmetry $\times$ blocks $[F(1,10)=6.74, p<.05]$ and appropriate individual comparisons $(p<.05)$ supported these observations. A significant interaction of task $\times$ reinforcement symmetry $\times$ retention interval $[F(2,20)=5.50, p<.05]$ was also found; this was caused by the fact that a significantly $(p<.05)$ higher hit rate occurred in DMTS than in DD tasks for symmetrical reinforcement at the 5-sec RI (pooled over baseline and test blocks).

\section{Proportion of False Alarms (Nonreinforced Leverpresses)}

False alarms in DMTS animals were nonreinforced responses to L-T and T-L pairs for matching to light $S_{1}$ and tone $S_{1}$, respectively. False alarms in DD animals were nonreinforced responses to T- $\mathrm{T}$ and $\mathrm{T}$ - $\mathrm{L}$ pairs combined for differentiation of light $S_{1}$ and nonreinforced responses to L-L, L-T pairs combined for differentiation of tone $S_{1}$. Changes in false alarms were clearly more consistent than changes in hits with increased RIs. Under all conditions, false alarms increased with increased RIs. Indeed, these increases were generally greater at 10- and 20 -sec RIs than at 5-sec RIs. A significant block $\times$ RI interaction $[F(2,20)=11.54, p<.01]$ and individual comparisons $(p<.01)$ confirmed these observations. Greater increases in false alarms to the visual than to the auditory $S_{1}$ occurred only for the DMTS group. A task $\times S_{1}$ modality $\times$ blocks interaction $[F(1,10)=7.77$, $p<.05]$ and individual comparisons $(p<.05)$ supported this observation. Furthermore, greater increases in false alarms were found in DMTS than in DD tasks only for matching to or differentiation of the visual $S_{1}$ (false alarms on T-T and T-L pairs in the DD animals). Reinforcement symmetry affected increases in false alarms to to the auditory $S_{1}$ primarily in the DMTS tasks. 


\section{DMTS}

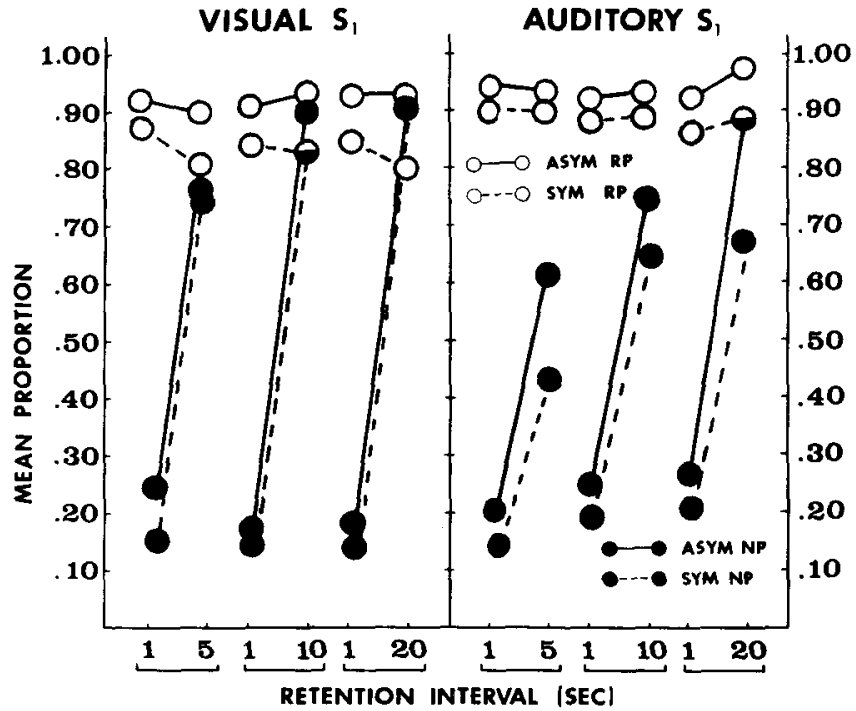

\section{DD}

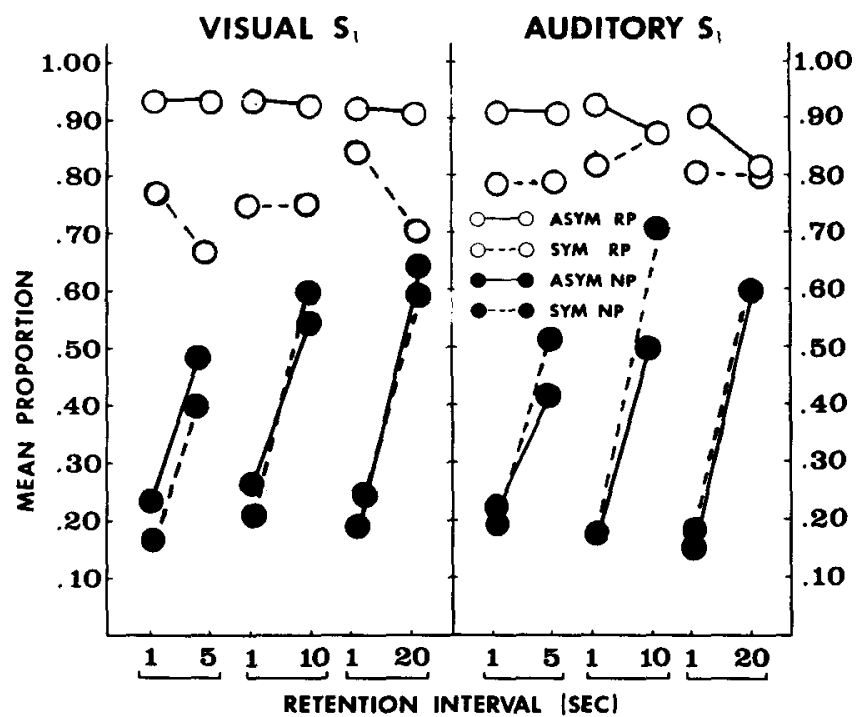

Figure 1. Mean proportions of hits or reinforced (RP) and false alarms or nonreinforced (NP) presses for matching to each sample stimulus $\left(S_{1}\right)$ in DMTS tasks or for differentiating each sample stimulus $\left(S_{1}\right)$ from the other in DD tasks during baseline (1-sec RI) and delay test (5-, 10-, 20-sec RIs) blocks in Experiment 1. ASYM = asymmetrically reinforced, and $S Y M=$ symmetrically reinforced form of each task.

As is evident in Figure 1 and from an interaction of task $\times S_{1}$ modality $\times$ reinforcement symmetry $\times$ blocks $[F(2,20)=4.94, p<.05]$, greater increases in false alarms to the tone $S_{1}$ occurred in the asymmetrically than in the symmetrically reinforced DMTS.

\section{$S_{1}$ Matching or Differentiation Scores, $P(\bar{A})$}

Figure 2 shows $P(\bar{A})$ scores for baseline (1-sec $\mathrm{RI})$ and delay test blocks (5-, 10-, and 20-sec RIs). $P(\bar{A})$ scores in DMTS indicate the ability of animals to successfully match $S_{2}$ to $S_{1}$ over various RIs. $P(\bar{A})$ scores in DD indicate the ability of animals to differentiate a light from tone $S_{1}$ when pressing to the light $S_{1}$ produced reinforcement (visual $S_{1}$ problem) or to differentiate a tone from light $S_{1}$ when pressing to the tone $S_{1}$ was reinforced (auditory $S_{1}$ problem). $P(\bar{A}) \mathrm{s}$ in the DMTS task for the visual $S_{1}$ were derived from proportions of hits to $\mathrm{L}-\mathrm{L}$ and false alarms to L-T pairs; $P(\bar{A})$ s for the auditory $\mathrm{S}_{1}$ were derived from proportions of hits to $T-T$ and false alarms to T-L pairs. $P(\bar{A}) \mathrm{s}$ in the DD task for the visual $\mathrm{S}_{1}$ were derived from proportions of hits to $\mathrm{L}-\mathrm{L}$ and $\mathrm{L}-\mathrm{T}$ pairs combined and false alarms to T-T and T-L pairs combined; $P(\bar{A}) \mathrm{s}$ for the auditory $\mathrm{S}_{1}$ were derived from proportions of hits to $\mathrm{T}-\mathrm{T}$ and $\mathrm{T}-\mathrm{L}$ pairs combined and false alarms to $\mathrm{L}-\mathrm{L}$ and $\mathrm{L}-\mathrm{T}$ pairs combined.

As shown in Figure 2, $P(\bar{A})$ scores generally declined on test blocks from baseline levels. $P(\bar{A})$ s declined more to the visual than to the auditory $S_{1}$ only in DMTS, however. Reinforcement symmetry influenced $P(\bar{A})$ scores most consistently in the DD group, where $P(\bar{A}) \mathrm{s}$ declined with increased RIs more in the symmetrically than in the asymmetrically reinforced problem. A similar effect was observed for the visual $S_{1}$ in DMTS at 5and 20-sec RIs. Comparisons between DMTS and DD tasks also revealed that declines in $P(\bar{A})$ were greater in the former than in the latter only for the visual $S_{1}$. A significant five-factor interaction $[F(2,20)=4.22, p<.05]$ was obtained. Individual comparisons generally supported our observations. Only observed declines in $P(\bar{A})$ at 5sec RI to the auditory $S_{1}$ in the asymmetrically reinforced DD failed to reach significance. In the DMTS task, $P(\bar{A}) \mathrm{s}$ declined significantly more to the visual than to the auditory $\mathrm{S}_{\mathbf{1}}(p<.05)$ except at the 5-sec RI in the asymmetrically reinforced version. No significant differences between visual and auditory $S_{1}$ differentiation were found in the DD tasks. Individual comparisons for the effects of reinforcement symmetry revealed that, in the DD task, $P(\bar{A})$ s declined to significantly lower levels $(p<.05)$ for symmetrically than for asymmetrically reinforced versions to either $S_{1}$, except in the case of the tone $S_{1}$ at the 20 -sec RI. Greater declines in $P(\widetilde{A})$ to symmetrically than to asymmetrically reinforced DMTS also occurred at the 5and 20-sec RIs for visual $S_{1}(p<.05)$. Individual comparisons between tasks also revealed that $P(\bar{A})$ s declined to significantly lower levels in the DMTS than in the DD task for the visual $S_{1}$ at each RI under either condition for reinforcement, but only for the auditory $S_{1}$ at the 5sec RI for symmetrical reinforcement and at the $20-\mathrm{sec}$ $\mathrm{RI}$ for asymmetrical reinforcement $(p<.05)$.

\section{Discussion}

Results from Experiment 1 generally confirmed predictions based on a retrospective/prospective account of working memory. Poorer delayed matching to visual than to auditory $S_{1}$ replicated previous findings (Cohen et al., 1984; Wallace et al., 1980) and suggest that rats cannot retrospect a visual $S_{1}$ as well as they can an auditory $S_{1}$. 


\section{DMTS}

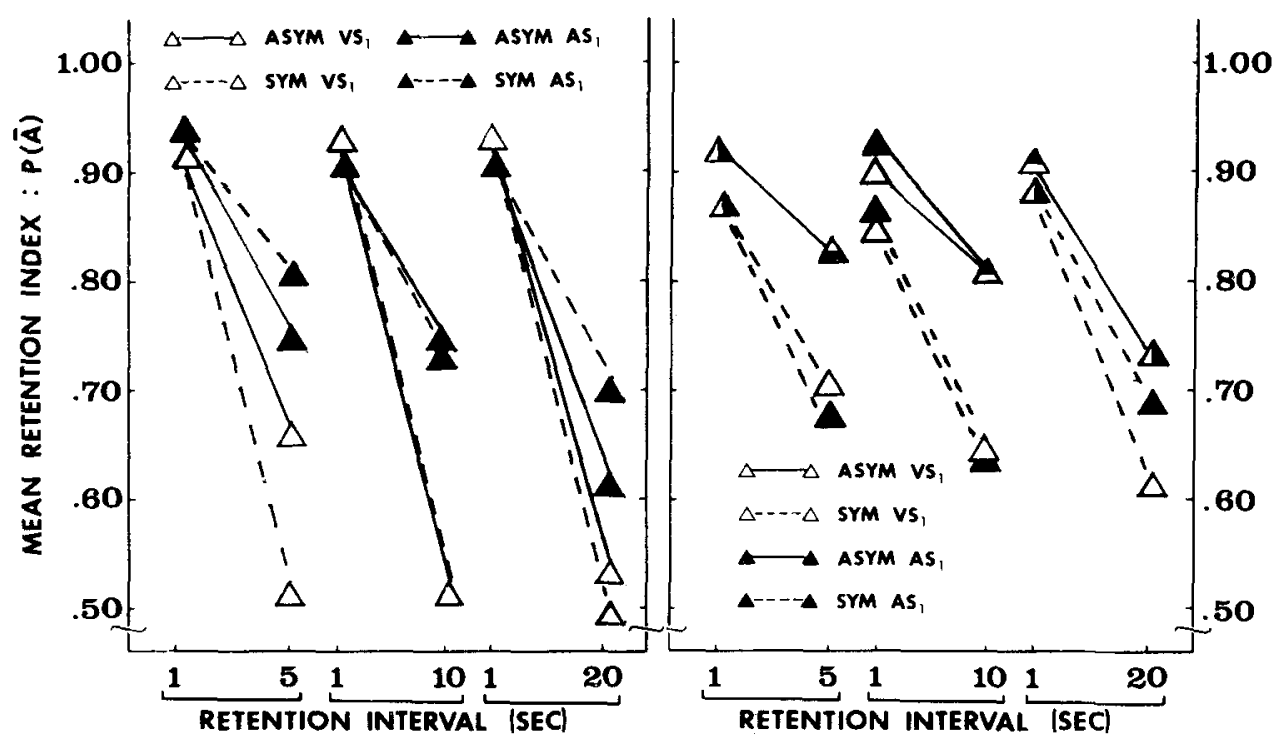

Figure 2. Mean signal sensitivity scores $[P(\bar{A})]$ for delayed matching to each sample stimulus in asymmetrically (ASYM) and symmetrically (SYM) reinforced DMTS, and for delayed differentiation of each sample stimulus in asymmetrically and symmetrically reinforced DD tasks during baseline (1-sec RI) and delay test (5-, 10-, 20-sec RIs) blocks in Experiment $1 . \mathrm{VS}_{1}=$ visual sample stimulus, $A S_{1}=$ auditory sample stimulus.

Complexity of the response rule affected performance more consistently in the DD task, as expected. Here, $P(\overline{\mathrm{A}}) \mathrm{s}$ declined from baseline levels more for symmetrical than for asymmetrical reinforcement. These findings suggest that animals found prospection of the more complex symmetrical reinforcement rule more difficult over longer RIs. Differences in delayed responding between variants of DD may not be due to differences in the amount of information provided by each $S_{1}$, but rather may be due to the contrast of such information. In the asymmetrically reinforced version, $S_{1}$ determines if reinforcement is possible, but in the symmetrically reinforced version, $S_{1}$ indicates only what type of response will be necessary for reinforcement. Introducing additional reinforcement expectancies of higher contrast for sample stimuli in the form of differential probabilities, amount, or kinds of rewards similarly improves actual or symbolic delayed matching performance in pigeons (Brodigan \& Peterson, 1976; DeLong \& Wasserman, 1981; Edwards, Jagielo, Zentall, \& Hogan, 1982; Honig, Matheson, \& Dodd, 1984).

Effects of reinforcement symmetry partially replicated those reported by Cohen et al. (1984). Although biases to leverpress with increased RIs were lower for symmetrical than for asymmetrical reinforcement in DMTS, such reductions were insufficient to attenuate declines in $P(\bar{A})$ to the tone $S_{1}$. Reinforcement symmetry did affect changes in $P(\bar{A})$ to the light $S_{1}$. Even though biases to respond to light $\mathrm{S}_{1}$ were lower for symmetrical reinforcement, $P(\bar{A}) \mathrm{s}$ declined more for symmetrical than for asymmetrical reinforcement. Since neither $S_{1}$ provided different expectancies for the possibility of reinforcement in DMTS, such effects of reinforcement symmetry on $P(\bar{A})$ scores to light $\mathrm{S}_{1}$ cannot be explained by the same processes in DD. Since $P(\bar{A})$ levels for either condition of reinforcement were not significantly higher than .50 , no retention, at $20-\mathrm{sec}$ RIs, these differences were more an artifact of reduction in hits (reinforced presses) without any compensatory attenuation in increases of false alarms (nonreinforced presses) for symmetrical reinforcement.

Although both groups received the same pairs of stimuli, they did not receive comparable training and testing procedures. DMTS animals received only one form of their task, whereas DD animals experienced a reversal of their initially acquired and tested discrimination. Delayed responding to each $S_{1}$ occurred within the same sessions for DMTS, but across sessions in DD. Proportions of hits and false alarms were also based on twice as many test trials within the same block of trials in the DD as in the DMTS task. These procedural differences may have contributed to differences in performance between these tasks. Associated with these problems was the fact that $P(\bar{A})$ scores were calculated from different stimulus pairs between each task. These possibly confounding factors prompted us to carry out the second experiment in which procedures between tasks were more comparable.

\section{EXPERIMENT 2}

\section{Method}

Design and Rationale

Experiment 2 was a systematic replication of the first experiment. In this experiment we wanted to determine if the type of task would 
alter the $S_{1}$ modality effect for the same subject trained and tested under more comparable conditions. All rats were trained and tested in symmetrically reinforced DMTS and DD tasks. Order of presentation of tasks was counterbalanced so that half the rats received training and testing first on DMTS then on DD, and half first on DD then on DMTS. When acquiring the DD task, half the rats were reinforced both for pressing to either $S_{2}$ following a light $S_{1}$ and for not pressing to either $S_{2}$ following a tone $S_{1}$; the other half were reinforced for pressing to either $S_{2}$ following a tone $S_{1}$ and for not pressing to either $S_{2}$ following a light $S_{1}$.

Testing for $S_{1}$ differentiation in DD was more comparable to testing for $S_{1}$ matching in DMTS in this experiment. Symmetrical reinforcement in which different responses to each $S_{1}$ were reinforced made it possible to compare differentiation between light and tone $S_{1}$ within the same block of sessions. To carry out such withinblock comparisons, we had to include both omissions and presses in definitions of hits and false alarms in the DD task. The outcome of presses was sufficient to determine hit and false-alarm rates in DMTS, however, due to the nature of that task. Light $S_{1}$ differentiation was determined from signal sensitivity $P(\bar{A})$ s derived from proportions of hits to $\mathrm{L}-\mathrm{L}$ and false alarms to $\mathrm{T}-\mathrm{L}$. Tone $\mathrm{S}_{1}$ differentiation was indicated by $P(\bar{A}) \mathrm{s}$ based on proportions of hits to T-T and false alarms to $L-T$. If pressing to light $S_{1}$ and not pressing to tone $S_{1}$ were reinforced, then hits were reinforced presses to $L-L$ and reinforced omissions to $T-T$; false alarms were nonreinforced presses to $T-L$ and nonreinforced omissions to $L-T$. If pressing to tone $S_{1}$ and not pressing to light $S_{1}$ were reinforced, hits were reinforced presses to $T-T$ and reinforced omissions to $L-L$; false alarms were nonreinforced presses to $\mathrm{L}-\mathrm{T}$ and nonreinforced omissions to T-L. $P(\bar{A})$ s for $S_{1}$ differentiation measured the subject's capacity to differentiate between sample stimuli for each type of response.

It should be noted that $P(\bar{A})$ for light $\mathrm{S}_{1}$ differentiation based on hits to all pairs with light $S_{1}(L-L, L-T)$ and false alarms to all pairs with tone $S_{1}(\mathrm{~T}-\mathrm{T}, \mathrm{T}-\mathrm{L})$ would be arithmetically identical to $P(\vec{A})$ for tone $\mathrm{S}_{1}$ based on the same combination of hits and false alarms. By using $P(\bar{A})$ for light $S_{1}$ based on proportions of L-L hits and T-L false alarms, and $P(\bar{A})$ for tone $\mathrm{S}_{1}$ based on proportions of T-T hits and L-T false alarms, we insured that each measure could vary independently. Other combinations of hits and false alarms could have been used to derive independent measures of $P(\bar{A})$ for each $S_{1}$, but we chose these combinations because (1), they allowed for a comparison between $S_{1}$ with $S_{2}$ held constant, and (2), they were most similar to combinations used to determine light and tone $S_{1}$ matching in DMTS.

By using the same stimulus pairs to obtain hit and false-alarm data in each task, we were also able to determine if the $S_{1}$ modality effect in DMTS was merely due to an artifact of calculating $P(\bar{A})$. The design of the first experiment did not permit such an investigation. In this experiment, however, we also recalculated $P(\bar{A})$ from DMTS based on hits from $\mathrm{L}-\mathrm{L}$ and false alarms from T-L, as in the DD task for light $S_{1}$ differentiation. Such $P(\bar{A})$ s were labeled light $S_{1}$ pseudodifferentiation in DMTS because the subject was not actually required to respond differently based on modality of $S_{1}$. $P(\bar{A})$ s from DMTS based on hits to T-T and false alarms to L-T were calculated to obtain tone $S_{1}$ pseudodifferentiation. If the $S_{1}$ modality effect in DMTS is merely a calculation artifact, then no difference between $S_{1}$ differentiation or pseudodifferentiation based on $S_{1}$ modality should be observed. In other words, animals should be able to detect $L-L$ from $T-L$ pairs as easily as T-T from L-T pairs in either task. If rats retain a light $S_{1}$ less well than a tone $S_{1}$, however, a stimulus modality effect will still be evident more in the DMTS than in the DD task. Better $S_{1}$ pseudodifferentiation for the light than for the tone $S_{1}$ should occur with increased RIs because of fewer false alarms to $T-L$ than to $L-T$ pairs.

By the same logic, we should also calculate pseudomatching $P(\bar{A}) \mathrm{s}$ for each $S_{1}$ in the DD task and compare them to actual matching $P(\bar{A})$ s in DMTS. Such reconstituted $P(\bar{A})$ s may not be able to be used, however, because hits and false alarms would be from different response modes in the DD task. If hits are from reinforced presses (omissions), then false alarms will be from nonreinforced omissions (presses) in each pseudomatching $P(\bar{A})$. Any bias to press would create better $P(\bar{A})$ scores when presses constituted hits rather than false alarms. Such effects would produce more variable and widely divergent pseudomatching $P(\bar{A})$ scores for DD than would matching $P(\bar{A})$ scores for DMTS.

\section{Subjects}

Eight male albino (Wistar) rats, approximately 150 days old at the start of this experiment, were used as subjects. These rats had acquired a compound light + tone successive discrimination in another experiment. That task was similar to the present discriminations, in that the animals were presented with a lever at fixed intervals to which they had to respond if it had been preceded by the compound stimulus. Because no retention intervals occurred between those stimulus-lever presentations, and because the rats responded similarly to each component of the compound in probe tests, we decided to use them in this experiment.

\section{Procedures}

The only difference between the present and the first experiment was that, in this experiment, all animals were trained and tested on symmetrically reinforced DMTS and DD tasks. Order of presentation of the tasks was counterbalanced so that half the rats received DMTS then DD and the other half had the order reversed. Half the animals acquired the symmetrically reinforced DD in which reinforcement was contingent upon leverpressing on $S_{2}$ following the light $S_{1}$ and not pressing on $S_{2}$ following the tone $S_{1}$. For the remaining animals, pressing to tone $S_{1}$ and omitting presses to light $S_{1}$ were reinforced. Otherwise, the training and testing procedures were the same as those described for Experiment 1.

\section{Data Analysis}

As in Experiment 1, proportions of hits and false alarms to specific pairs of stimuli were calculated for each block of baseline and retention test sessions. In the DMTS task, reinforced presses to L-L and T-T were identified as hits; false alarms were nonreinforced presses to $\mathrm{L}-\mathrm{T}$ and $\mathrm{T}-\mathrm{L}$ pairs. In the DD task, hits were reinforced presses to $\mathrm{L}-\mathrm{L}$ and reinforced omissions to $\mathrm{T}-\mathrm{T}$; false alarms were nonreinforced presses to $T-L$ and nonreinforced omissions to $L-T$ for animals trained to press to light $S_{1}$ and withhold pressing to tone $S_{1}$. For animals trained with the opposite rule, hits were reinforced presses to $\mathrm{T}-\mathrm{T}$ and reinforced omissions to $\mathrm{L}-\mathrm{L}$; false alarms were nonreinforced presses to $\mathrm{L}-\mathrm{T}$ and nonreinforced omissions to $\mathrm{T}-\mathrm{L}$.

$P(\bar{A})$ scores for matching to the light $\mathrm{S}_{\mathbf{1}}$ in DMTS were based on proportions of hits and false alarms to $\mathrm{L}-\mathrm{L}$ and $\mathrm{L}-\mathrm{T}$, respectively. $P(\bar{A})$ s for matching to tone $S_{\mathbf{1}}$ in DMTS were derived from proportions of hits and false alarms to $\mathrm{T}-\mathrm{T}$ and $\mathrm{T}-\mathrm{L}$, respectively. In the DD task, $P(\bar{A})$ s for light $\mathrm{S}_{1}$ differentiation were calculated from proportions of hits and false alarms to $\mathrm{L}-\mathrm{L}$ and $\mathrm{T}-\mathrm{L}$, respectively; $P(\bar{A})$ s for tone $S_{1}$ differentiation came from proportions of hits and false alarms to T-T and L-T pairs, respectively. A set of supplementary $P(\bar{A})$ s from DMTS data was calculated with the same combination of hits and false alarms as in DD. Light $S_{1}$ pseudodifferentiation in DMTS was determined from $P(\bar{A}) \mathrm{s}$ based on proportions of hits and false alarms to $\mathrm{L}-\mathrm{L}$ and $\mathrm{T}-\mathrm{L}$ pairs, respectively; $P(\bar{A})$ s for tone $S_{1}$ pseudodifferentiation were based on proportions of hits and false alarms to $T-T$ and $L-T$ pairs, respectively.

Arcsin transformations of proportions of hits and false alarms and of $P(\bar{A})$ s were analyzed by appropriate ANOVAs and NewmanKeuls tests for individual comparisons.

\section{Results \\ Proportion of Hits and False Alarms}

Figure 3 shows proportions of hits and false alarms at each baseline (1-sec RI) and test block (5-, 10-, and 20$\sec \mathrm{RI}$ ) in each task. Although false alarms appeared to 


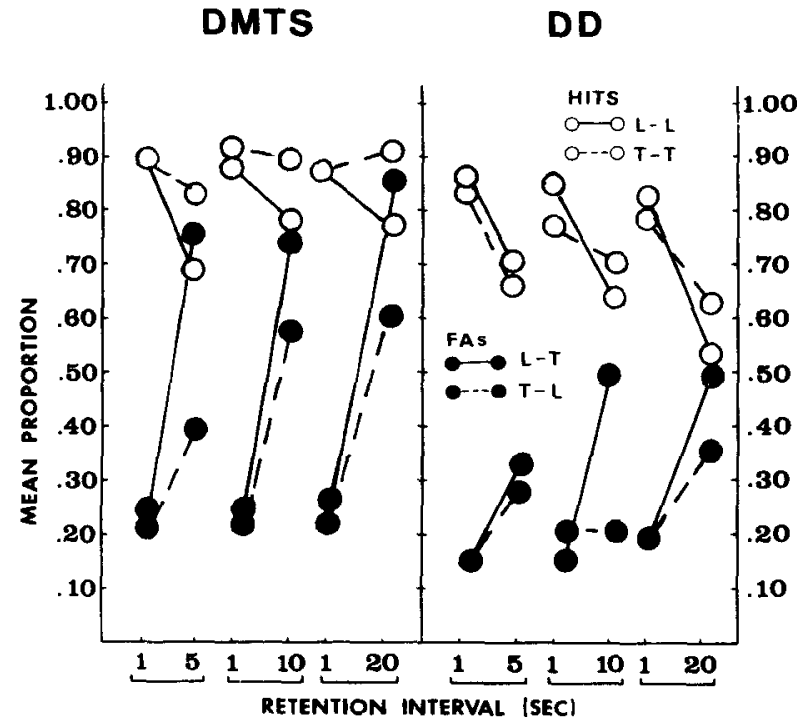

Figure 3. Mean proportion of hits to light-light ( $L-L)$ and tonetone (T-T) pairs; mean proportion of false alarms to light-tone ( $\mathrm{L}$ $T$ ) and tone-light (T-L) pairs in DMTS and DD tasks during baseline (1-sec RI) and delay test (5-, 10-, 20-sec RIs) blocks in Experiment 2.

increase more from baseline at each test RI and hits declined less in DMTS than in DD, these differences are a measurement artifact. In DMTS, hits and false alarms were defined exclusively in terms of leverpress outcomes, but in DD they were defined in terms of both omissions and press outcomes. Since a strong bias to press still persisted in symmetrically reinforced discrimination, as shown in Experiment 1 and by Cohen et al. (1984), omissions could have enhanced declines in hits and attenuated increases in false alarms to produce the observed group averages in the DD task. To test this possibility, a preliminary comparison between changes in presses and omissions for hits and false alarms in the DD task was carried out by separate ANOVAs of response mode $x$ blocks $\times$ RI for hits and false alarms. As expected, response mode $\times$ blocks interactions were found for hits $[F(1,14)=$ $43.07, p<.01]$ and false alarms $[F(1,14)=28.80$, $p<.01]$, which were attributed solely to decreases in reinforced omissions and increases in nonreinforced presses from baseline to test levels. In view of these effects, we carried out separate $S_{1}$ modality $\times$ blocks $\times$ RI ANOVAs for hits and false alarms for each task and compared only differences in the patterns of change between tasks as a function of $S_{1}$ modality.

Proportion of hits. As shown in Figure 3, $S_{1}$ modality affected declines in hits from baseline levels only in the DMTS task. Here, hits declined slightly, but reliably, more to light than to tone $S_{1}$. A significant $S_{1}$ modality $\times$ blocks $[F(1,7)=6.17, p<.05]$ interaction occurred. Individual comparisons indicated that only hits to light $\mathrm{S}_{1}$ significantly declined from baseline levels $(p<.01)$ and to levels significantly lower than those for tone $S_{\mathbf{1}}$ at test blocks $(p<.05)$. In the DD task, a main effect for blocks $[F(1,7)=153.30, p<.001]$ and individual comparisons confirmed that hits to either $\mathbf{S}_{\mathbf{1}}$ declined significantly on test blocks $(p<.01)$ to equivalent levels.

Proportion of false alarms. Figure 3 shows that false alarms increased more to the light $S_{1}$ (L-T pairs) than to tone $S_{1}$ (T-L pairs) in either task. An interaction for $S_{1}$ modality $\times$ blocks $\times$ RI was found in DMTS $[F(2,14)$ $=5.20, p<.05]$ and $\mathrm{DD}[F(2,14)=4.25, p<.05]$. Although false alarms increased significantly to each $S_{1}$ in the DMTS task, they rose significantly more to the light $S_{1}$ than to the tone $S_{1}$ at each RI $(p<.05)$. False alarms also increased more at the 20 - than at either the 10 - or 5-sec RI for light $S_{1}$ and more at the 10- than at the 5-sec $\mathrm{RI}$ for tone $S_{1}$. In the DD task, false alarms increased significantly only to the light $S_{1}$ at the 10 - and 20 -sec RIs $(p<.01)$ and to levels significantly above those to the tone $\mathrm{S}_{1}(p<.05)$.

$S_{1}$ matching or differentiation scores, $P(\bar{A})$. Figures 4 and 5 show $P(\bar{A})$ scores for each baseline (1-sec RI) and delay test block (5-, 10-, and 20-sec RI) for each task. In Figure 4, $P(\bar{A})$ s for DMTS determine matching to each $\mathrm{S}_{1} ; P(\bar{A}) \mathrm{s}$ for DD determine differentiation of each $\mathrm{S}_{1}$. In Figure 5, $P(\bar{A})$ s for DMTS were based on recalculations of the same combinations of hit and false-alarm proportions as were those for DD. These $P(\bar{A}) \mathrm{s}$, in Figure 5, for DMTS are termed $S_{1}$ pseudodifferentiation $P(\bar{A})$ s.

Prior to carrying out ANOVAs for direct comparisons between tasks, we had to determine if using different response measures to generate $P(\bar{A})$ s in DD affected this

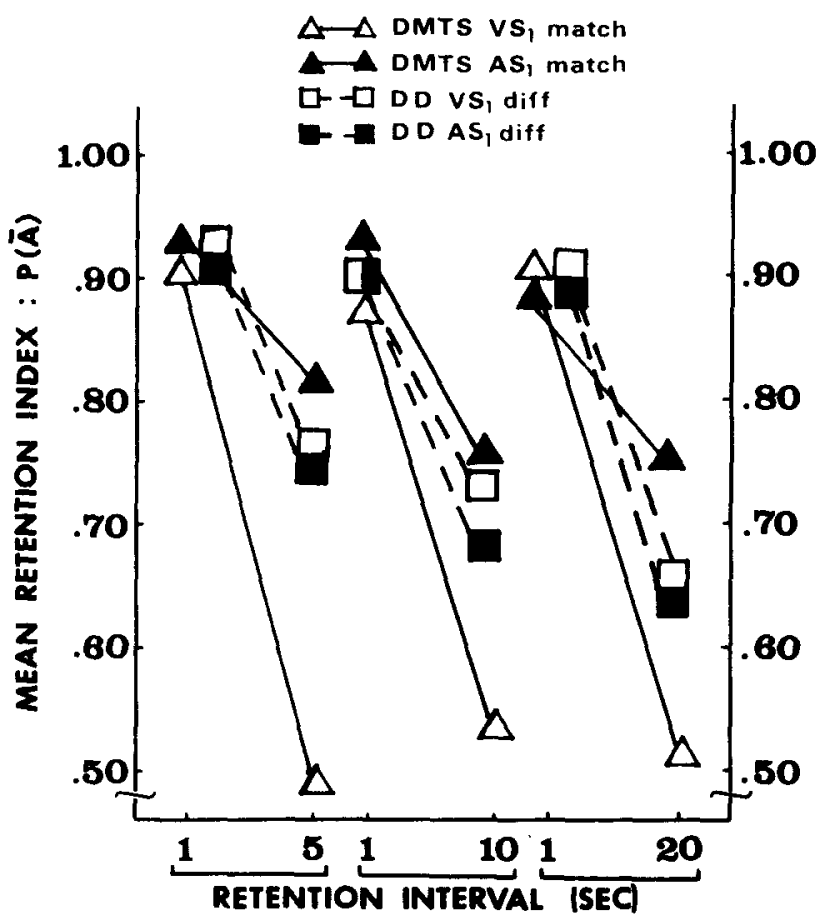

Figure 4. Mean signal sensitivity scores $[P(\bar{A})]$ for delayed matching (match) to visual $\left(\mathrm{VS}_{1}\right)$ and auditory $\left(\mathrm{AS}_{1}\right)$ sample stimuli in DMTS and for delayed differentiation (diff) to each $S_{1}$ in DD during baseline (1-sec RI) and delay test (5-, 10-, 20-sec RIs) blocks in Experiment 2. 


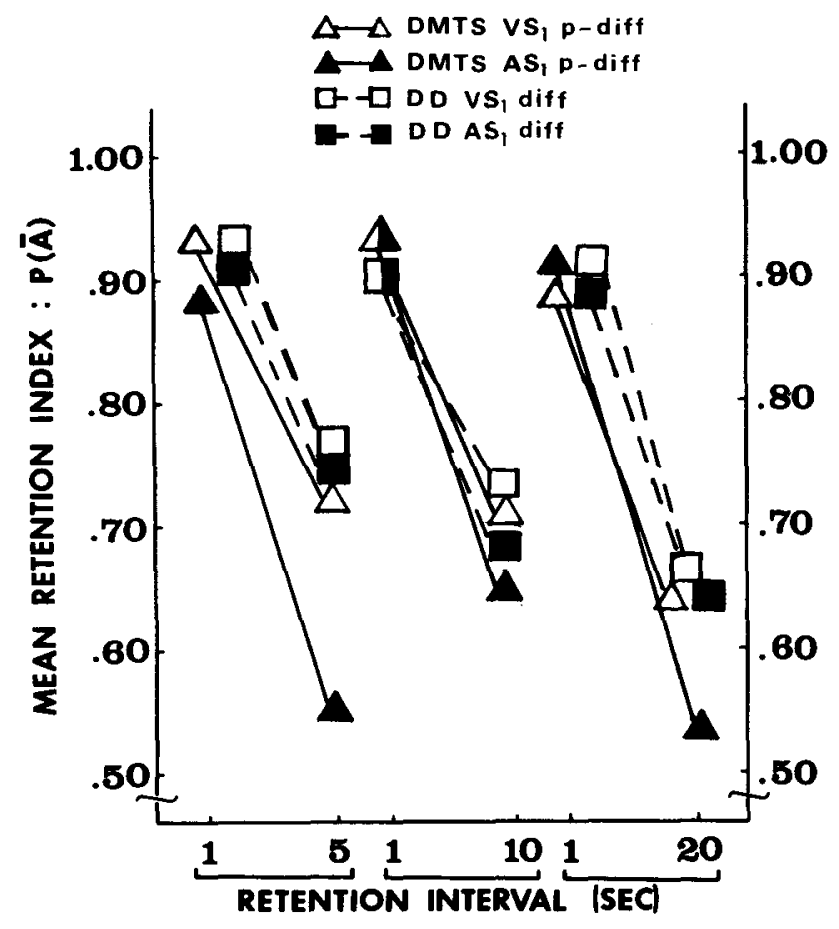

Figure 5. Mean signal sensitivity scores $[P(\bar{A})]$ for delayed pseudodifferentiation (p-diff) in DMTS and delayed differentiation (diff) in $\mathrm{DD}$ to visual $\left(\mathrm{VS}_{1}\right)$ and auditory $\left(\mathrm{AS}_{1}\right)$ sample stimuli during baseline (1-sec RI) and delay test (5-, 10-, 20-sec RIs) blocks in Experiment 2.

measure. It will be recalled that if an animal had $P(\bar{A}) \mathrm{s}$ generated from presses for one $\mathrm{S}_{1}$, its $P(\bar{A})$ s for the other $\mathrm{S}_{1}$ were based on omissions. Preliminary Mann-Whitney $U$ comparisons between $P(\bar{A})$ s generated from presses and those based on omissions failed to reveal any significant differences at each block of sessions. Pseudomatching to sample $P(\bar{A}) \mathrm{s}$ in DD could not be used, however, because different combinations of presses and omissions would have had to be used in calculating $P(\bar{A})$ s. $P(\bar{A})$ s would have had to be based on proportions of reinforced presses and nonreinforced omissions or on proportions of reinforced omissions and nonreinforced presses. The former combination would be expected to produce greater $P(\bar{A}) \mathrm{s}$, especially during delay test blocks due to a bias to leverpress. Preliminary comparisons between both types of $P(\bar{A})$ s at each block (Mann-Whitney $U$ tests) revealed that $P(\bar{A})$ s based on proportions of reinforced presses and nonreinforced omissions were significantly higher than $P(\bar{A}) \mathrm{s}$ from proportions of reinforced omissions and nonreinforced presses at each test block $(U \geq 9, p \leq .01) . P(\bar{A})$ transformed data were analyzed by four-way ANOVAs (task $\times S_{1}$ modality $\times$ blocks $\times \mathrm{RI}$ ), with all factors repeated and Newman-Keuls tests for individual comparisons.

Inspection of Figure 4 shows that although $P(\bar{A}) \mathrm{s}$ declined from baseline levels at delay test blocks in each task, such declines were greater to the visual than to the auditory $S_{1}$ only in DMTS for matching-to-sample performance. Delayed discriminative responding was not uniformly better than delayed matching performance. $P(\bar{A}) \mathrm{s}$ for matching declined more in DMTS to visual $S_{1}$ but less to the auditory $\mathrm{S}_{1}$ than did $P(\bar{A})$ s to either $\mathrm{S}_{1}$ in DD. A significant interaction of task $\times S_{1}$ modality $\times$ blocks $[F(1,7)=72.01, p<.01]$ and individual comparisons $(p<.01)$ confirmed these observations.

When $P(\bar{A})$ s for $\mathrm{S}_{1}$ pseudodifferentiation in DMTS were compared to $P(\bar{A}) \mathrm{s}$ for actual $\mathrm{S}_{1}$ differentiation in Figure $5, S_{1}$ modality effects were again more prevalent in DMTS than DD. $P(\bar{A})$ s declined more for tone than for light $S_{1}$ pseudodifferentiation at 5- and 20-sec RIs. A similar difference at 10 -sec RI was seen in the DD task. Although a four-factor interaction barely missed significance $[F(2,14)=3.15, p=.074]$, we ran individual comparisons anyway to confirm our observations $(p<.05)$.

\section{Discussion}

Results in the second experiment, with more comparable testing procedures between tasks, replicated the findings from the first experiment. Modality of $S_{1}$ affected delayed responding primarily in DMTS rather than in DD for the same rat. Delayed matching declined more to a visual $S_{1}$ than to an auditory $S_{1}$, a finding similar to that reported in earlier research (Cohen et al., 1984; Wallace et al., 1980). That such an effect was largely absent in DD cannot be attributed to differences in calculating $P(\bar{A})$. When $P(\bar{A})$ s in DMTS were recalculated as in DD, $S_{1}$ modality was still more influential in affecting declines in these scores in the DMTS than in the DD task. Here $S_{1}$ pseudodifferentiation was poorer to tone than to light $S_{1}$ because more false alarms occurred to $L-T$ than to $T$ $L$ pairs. Poorer retention of the light $S_{1}$ in DMTS accounts for these differences in $S_{1}$ pseudodifferentiation in DMTS.

Although retention of response rules varying in complexity were not compared in this experiment, our findings also indicate that performance in DD may not always be superior to that in DMTS. Under a relatively complex response rule for symmetrical reinforcement, DD performance declined more for tone $S_{1}$ but less for light $S_{1}$ than did DMTS performance. These findings are contrary to earlier reports of better DD than DMTS performance at increased delays (Honig \& Dodd, 1983; Honig \& Wasserman, 1981; Smith, 1967) but are not inconsistent with the dual-process theory (Honig \& Thompson, 1982).

Although our findings point to a predominant retrospective process in DMTS and a prospective one in DD, neither may be exclusively promoted by each task. Experiment 2 allowed for a more refined analysis of $S_{1}$ modality effects in DD through measures of responding to each stimulus pair. $S_{1}$ modality affected changes in both hits and false alarms in DMTS, as expected, but $S_{1}$ modality also influenced changes in false alarms in the DD task. False alarms increased more to $\mathrm{L}-\mathrm{T}$ than to $\mathrm{T}-\mathrm{L}$ pairs 
in DD. Such differences were not large enough to affect $P(\vec{A})$ s, but they do raise the possibility that some degree of retrospection of $S_{1}$ also occurred in the DD task.

\section{EXPERIMENT 3}

The fact that the $S_{1}$ modality effect was found only in DMTS in the first two experiments was considered as evidence for retrospective memory in DMTS and prospective memory in DD. One might argue, however, that prospection could also account for the modality effect in DMTS, if rats are less able to maintain an instruction from a sample stimulus to respond to a visual than they are to an auditory test stimulus. Consequently, the modality of the appropriate $S_{2}$ for which a response will provide reinforcement, rather than the modality of the initial sample stimulus, should affect actual or symbolic matching. If a tone $S_{1}$ indicates that reinforcement is contingent upon responding when $S_{2}$ is a light rather than a tone, and light $S_{1}$ indicates the opposite contingency, then according to this prospective account, accuracy of delayed responding should be poorer following the tone than following the light $S_{1}$. According to the retrospective account, accuracy of delayed responding to $S_{2}$ should still be poorer after the light than after the tone $S_{1}$. We tested these alternative accounts in the third experiment by comparing the performance of rats trained and tested on asymmetrically reinforced DMTS with that of rats trained and tested on an asymmetrically reinforced delayed mismatchingto-sample (DMmTS) discrimination. In the latter task, reinforcement was contingent upon responding to $S_{2}$ when its modality differed from $S_{1}$ (L-T, T-L pairs).

\section{Method}

\section{Subjects}

The subjects were 14 male albino rats that were veterans of the same compound discrimination task as those in the first two experiments. The animals were randomly and equally divided into the DMTS and DMmTS groups.

\section{Procedure}

The basic procedures for training and testing were the same as those described for the initial experiments. All animals were trained and tested on the asymmetrically reinforced versions of their assigned task. Reinforcement was contingent for pressing on $S_{\mathbf{2}}$ only when it matched $S_{1}$ (L-L, T-T) in DMTS and only when it differed from $S_{1}(L-T, T-L)$ in DMmTS. Correct omissions were not reinforced in either task. Three-way ANOVAs [task (DMTS vs. DMmTS) $\times$ blocks (baseline vs. delay tests) $\times \operatorname{RI}(5,10,20 \mathrm{sec})]$, with repeated measures on the last two factors and Newman-Keuls tests for individual comparisons, were carried out on arcsin transformations of proportions of hits (reinforced presses), proportions of false alarms (nonreinforced presses), and $P(\bar{A})$ retention scores.

\section{Results}

\section{Proportions of Hits and False Alarms}

Figure 6 shows proportions of hits and false alarms at each baseline (1-sec RI) and test block (5-, 10-, and 20sec RIs) in each task. Hits declined slightly more to the light than to the tone $S_{1}$ in each task, as shown in Figure 6 and found by a significant $S_{1}$ modality $\times$ blocks interac-
DMTS

DMmTS

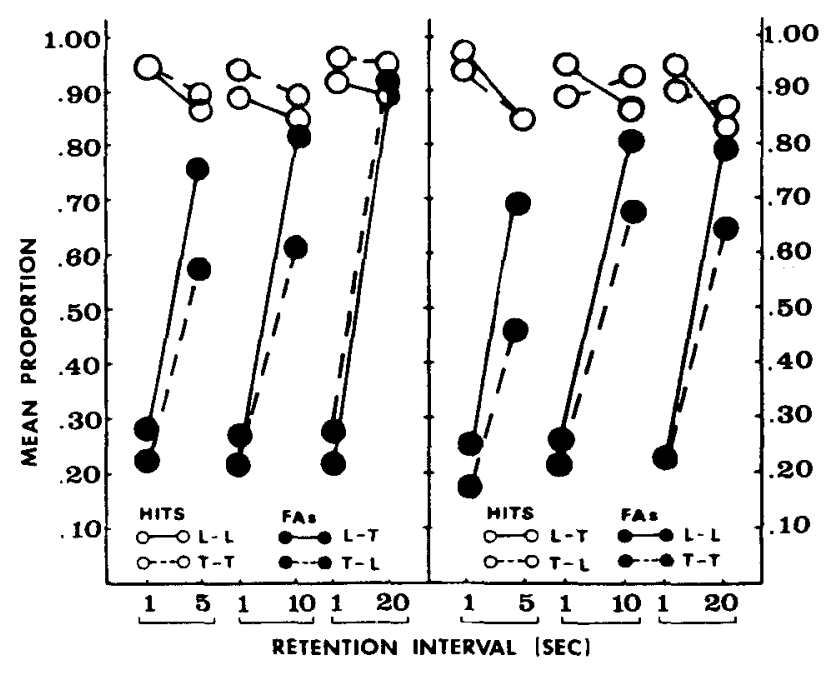

Figure 6. Mean proportion of hits and false alarms for delayed matching (DMTS group) or mismatching (DMmTS group) to the visual $\left(V_{S_{1}}\right)$ and auditory $\left(A S_{1}\right)$ sample stimuli during baseline (1sec RI) and delay test (5-, 10-, 20-sec RIs) blocks in Experiment 3.

tion $[F(1,12)=17.27, p<.01]$. A significant triple interaction of these two factors with task was also uncovered $[F(1,12)=7.05, p<.05]$, but this was only due to significantly more hits made to light than to tone $S_{1}$ at baseline in DMTS $(p<.05)$.

False alarms increased substantially from baseline at each delay, but did so more to the light than to the tone $S_{1}$ in each task. A significant interaction between $S_{1}$ modality and blocks $[F(1,12)=11.56, p<.01]$ supported this observation. Individual comparisons further revealed that false alarms significantly increased $(p<.05)$ more to light than to tone $S_{1}$ except at the 20-sec RI in the DMTS task.

\section{$S_{1}$ Matching or Mismatching Scores, $P(\bar{A})$}

Figure 7 shows $P(\bar{A})$ scores from baseline (1-sec RI) and delay test blocks (5-, 10-, and 20-sec RIs). Within each task, $P(\bar{A})$ s declined from baseline levels more to the light than to the tone $S_{1}$. A significant interaction between $S_{1}$ modality and blocks $[F(1,12)=67.58, p<.01]$ and individual comparisons $(p<.01)$ supported this observation. No significant effects due to task were found.

\section{Discussion}

Aside from two minor differences in changes in hits and false alarms, animals in each task behaved similarly by increasing false alarms and decreasing hits and $P(\bar{A})$ scores more from baseline levels to light than to tone $S_{1}$. These results clearly support a retrospective rather than a prospective interpretation of the $S_{1}$ modality effect in DMTS observed in the first two experiments. These findings also replicate and extend those of Wallace et al. (1980), who found better simultaneous delayed symbolic 
DMTS

DMmTS

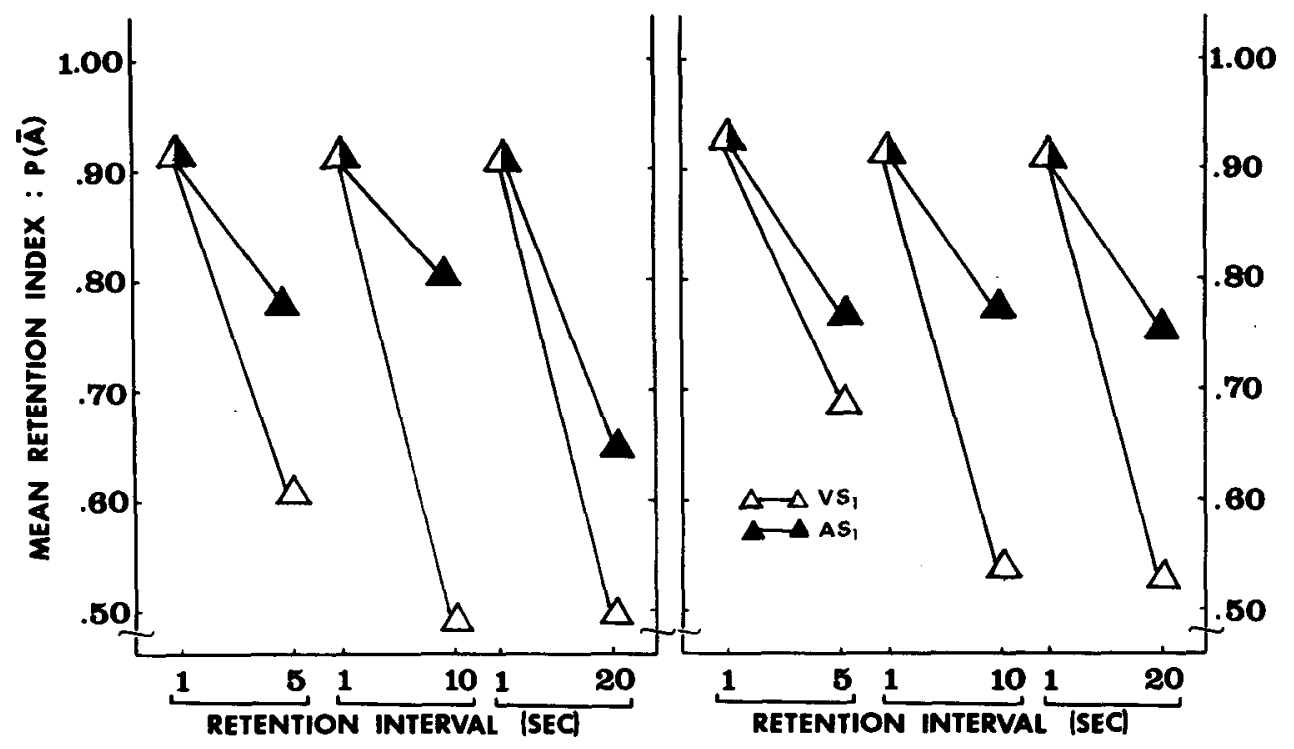

Figure 7. Mean signal sensitivity scores $[P(\bar{A})]$ for delayed matching (DMTS group) or mismatching (DMmTS group) to the visual (VS $)_{1}$ and auditory $\left(\mathrm{AS}_{1}\right)$ sample stimuli during baseline (1-sec RI) and delay test (5-, 10-, 20-sec RIs) blocks in Experiment 3.

matching to sample for a tone than for a light $S_{1}$ (Experiment 1). In their experiment, however, the animals had to choose between two discrete visual stimuli (steady vs. flashing light). The global visual $S_{1}$ may have proactively interfered with choices between the discrete, localized visual stimuli. Our design, which used the same test as sample stimuli in both an actual matching and a symbolic matching (mismatching) procedure, eliminated the possibility of differential $S_{1}$ proactive interference.

\section{GENERAL DISCUSSION}

Findings from the first two experiments are consistent with Honig and Thompson's (1982) dual-process theory of working memory. In DMTS, the animal must generally retrospect the nature of $S_{1}$ to perform correctly to $S_{2}$, but in DD, $S_{1}$ provides the animal with all the information to respond correctly to $S_{2}$, so that the animal only has to prospect or rehearse the instruction about responding signaled by $S_{1}$. Therefore, differences in the ability to retrospect different $S_{1} s$ in DMTS should disappear in DD. Rats in the present study appeared to retrospect a visual sample stimulus less well than an auditory sample stimulus, but prospected the response rules indicated by each equally well or poorly over increased retention intervals. Prospection may lead to either better or poorer performance than retrospection, however, depending upon the complexity of information to be retained under each process.

Findings from the third experiment indicate that the $S_{1}$ modality effect observed in DMTS cannot be accounted for by prospection-that is, animals having more difficulty remembering to press on a light $S_{2}$ than on a tone $S_{2}$ as signaled by $S_{1}$. In Experiment 3, rats were equally less able to delay matching or mismatching to a visual than to an auditory $S_{1}$.

Even though retrospection appears to be dominant in DMTS and prospection appears to be dominant in DD, each process may not be exclusively associated with each task. Some evidence for retrospection in DD was observed in Experiment 2, in which rats increased false alarms more to the visual than to the auditory $S_{1}$. Perhaps such an $S_{1}$ modality effect indicates residual retrospection, initially used in early acquisition, but not yet completely supplanted by prospection. A similar idea was proposed by Weisman, Wasserman, Dodd, and Larew (1980), who found that control by $S_{2}$ declined in favor of control by stimulus order in later training on stimulus sequence discrimination in pigeons. Some residual retrospection of $S_{1}$ during increased retention intervals also may have been maintained by our rats in Experiment 2 to retrieve complex instructions initiated by that stimulus. In other words, retrospection may serve as an aid for prospective retention or rehearsal in DD tasks.

In a rather obvious way, prospection must also occur in DCD tasks. Although the subject may have to retrospect $S_{1}$ until $S_{2}$ occurs, when $S_{2}$ is presented, the rat, for accurate performance, will have to prospect the response instruction and outcome associated with the stimulus sequence. Evidence of this type of temporal sequencing of each process is found in greater disruption of matching accuracy by increased RIs following $S_{1}$ than following $S_{2}$ (Honig \& Wasserman, 1981; Weisman \& DiFranco, 1981). If the rule to be prospected during post-S $S_{2}$ RIs is 
sufficiently complex, however, such general differences in performance would not always occur. In our preparation, for example, increasing RI only after $S_{1}$ rather than after $S_{2}$ should produce poorer matching to visual than to auditory $S_{1}$. If, as Honig (1978) suggests, a response to terminate a present trial and advance to the next trial indicates prospective anticipation of a negative outcome, then when the subject chooses to make an advance response within a DCD task should also indicate possible retrospection. Weisman, Gibson, and Rochford (1984) found that pigeons would advance to the next trial at $S_{1}$ when it signaled a negative outcome, but waited to advance on $S_{2}$ when such outcomes were codetermined by both stimuli. Delaying advance responding until $S_{2}$ may require some retrospection of $S_{1}$.

A more difficult question arises if prospection as well as retrospection occurs to $S_{1}$ in DCD tasks. The design of the present study was not suited to assess such possible simultaneous processing. Enhancing prospection of $S_{1}$ by having it differentially predict some aspect of reinforcement, as discussed earlier, or by requiring subjects to respond differently to each $S_{1}$ to produce test stimuli (Shimp \& Moffitt, 1977, Experiment 3; Urcuioli \& Honig, 1980; Zentall, Hogan, Howard, \& Moore, 1978) improves delayed actual or symbolic matching in the pigeon. The general interpretation of these findings has been that such improvements represent a usurpation of a less efficient retrospective process by a prospective one. As we have shown, however, prospection does not necessarily guarantee better retention but will eliminate differential retention based on stimulus modality. Therefore, a better indication of reduction of retrospection by such experimentally induced prospection would be a decline of the stimulus-modality effect. Such a demonstration still does not determine if prospection of $S_{1}$ occurs naturally in DCD tasks. If prospection represents slower instructional set learning and retrospection represents an initial memory process, then our techniques of differentiating retention to light and tone $S_{1}$ could be used to assess the development of acquiring response rules. We might expect that the stimulus modality effect should diminish or possibly disappear (if prospection takes over completely) with continued DCD training.

The preceding discussion offers some possible ways to study the relative contribution of retrospective and prospective processes in short-term retention. Those ideas rest upon the demonstration that some kinds of stimuli will be retained better than others. Although humans share with rats a similar characteristic of being able to retain information presented auditorily better than they can information presented visually (Conrad \& Hall, 1968; Murdock, 1968; Murdock \& Walker, 1969; Watkins, Watkins, \& Crowder, 1974), pigeons fail to display such a distinction (Kraemer \& Roberts, 1984). Perhaps the demonstration of differential short-term retention of sample stimuli based on other orthogonal dimensions may have to be established before similar investigations are carried out on pigeons. Kraemer and Roberts's (1984) findings do raise the possibility that separate modalityspecific memory areas, each possessing different decay, interference, and retrieval characteristics, may be more a mammalian than an avian trait. Finally, we note that Kraemer and Roberts's (1984) procedures differed from ours in that they trained and tested their pigeons with stimuli exclusively on one modality and then exclusively on the other. We presented both types of stimuli within the same task. A replication of their work should be carried out with rats to determine if modality-specific effects on $S_{1}$ retention are influenced by training and testing procedures.

\section{REFERENCES}

BLovGH, D. S. (1959). Delayed matching in the pigeon. Journal of the Experimental Analysis of Behavior, 1, 151-160.

Brodigan, D. L., \& Peterson, G. B. (1976). Two-choice discrimination performance of pigeons as a function of reward expectancy, prechoice delay, and domesticity. Animal Learning \& Behavior, 4, 121-124.

Cohen, J. S., Escotr, M., \& Ricciardi, P. (1984). The role of reinforcement symmetry and stimulus modality in successive delayed matching to sample in the rat. Canadian Journal of Psychology, 38, 63-79.

COHEN, J. S., \& Fuerst, D. Nonparametric signal sensitivity vs. dis crimination indices of sample stimulus retention in the rat. Paper presented at Conference on Animal Learning, Dalhousie University, Halifax, June 1985

ConRad, R., \& Hall, A. J. (1968). Input modality and the serial position curve in short-term memory. Psychonomic Science, 10, 135-136.

D'Aмато, M. R. (1970). Experimental psychology: Methodology, psychophysics, and learning. New York: McGraw-Hill.

DeLong, R. E., \& Wasserman, E. A. (1981). Effects of differential reinforcement expectancies on successive matching-to-sample performance in pigeons. Journal of Experimental Psychology: Animal Behavior Processes, 7, 394-412.

Edwards, C. A., Jagielo, J. A., Zentall, T. R., \& Hogan, D. E. (1982). Acquired equivalence and distinctiveness in delayed matching to sample by pigeons: Mediation by reinforcer-specific expectancies. Journal of Experimental Psychology: Animal Behavior Processes, 8, 248-259.

Ferguson, G. A. (1981). Statistical analysis in psychology and education (5th ed.). New York: McGraw-Hill.

GRANT, D. S. (1976). Effect of sample presentation time on long-delay matching in the pigeon. Learning \& Motivation, 1, 580-590.

HonIG, W. K. (1978). Studies of working memory in the pigeon. In S. H. Hulse, H. Fowler, \& W. Honig (Eds.), Cognitive processes in animal behavior (pp. 211-248), Hillsdale, NJ: Erlbaum.

HoNIG, W. K., \& DoDD, P. W. D. (1983). Delayed discrimination in the pigeon: The role of within-trial location of conditional cues. Animal Learning \& Behavior, 11, 1-9.

Honig, W. K., Matheson, W. R., \& Dodd, P. W. D. (1984). Outcome expectancies as mediators for discriminative responding. Canadian Journal of Psychology, 38, 196-217.

HoniG, W. K., \& Thompson, R. K. R. (1982). Retrospective and prospective processing in animal working memory. in G. H. Bower (Ed.), The psychology of learning and motivation (Vol. 16, pp. 167197). New York: Academic Press.

Honig, W. K., \& Wasserman, E. A. (1981). Performance of pigeons on delayed simple and conditional discriminations under equivalent training conditions. Learning \& Motivation, 12, 149-170.

KoNORSKI, J. (1959). A new method of physiological investigation of recent memory in animals. Bulletin de L'Academie Polonaise des Sciences, 7, 115-119.

Kraemer, P. J., \& RoberTs, W. A. (1984). Short-term memory for visual and auditory stimuli in pigeons. Animal Learning \& Behavior, 12, 275-284. 
MCNicol, D. (1972). A primer of signal detection theory. London: Allen \& Unwin.

MURDOCK, B. B., JR. (1968). Modality effects in short-term memory Storage or retrieval? Journal of Experimental Psychology, 77, 79-86.

MURDOCK, B. B., JR., \& WALKER, K. D. (1969). Modality effects in free recall. Journal of Verbal Learning \& Verbal Behavior, 8, 665-676.

SHImP, C. P., \& MoffitT, M. (1977). Short-term memory in the pigeon: Delayed-pair-comparison procedures and some results. Journal of the Experimental Analysis of Behavior, 28, 13-25.

SMITH, L. (1967). Delayed discrimination and delayed matching in pigeons. Journal of the Experimental Analysis of Behavior, 10, $529-533$.

Swets, J. A., Tanner, W. P., Jr., \& Birdsall, T. G. (1961). Decision processes in perception. Psychological Review, 68, 301-340.

TANneR, W. P., JR., \& Swets, J. A. (1954). A decision-making theory of visual detection. Psychological Review, 61, 401-409.

Urcuioli, P. J., \& Honig, W. K. (1980). Control of choice in conditional discriminations by sample-specific behaviors. Journal of Experimental Psychology: Animal Behavior Processes, 6, 251-277.

Wallace, J., Steinert, P. A., Scobie, S. R., \& SPEar, N. E. (1980) Stimulus modality and short-term memory in rats. Animal Learning \& Behavior, 8, 10-16.

Watkins, M. J., Watkins, O. G., \& Crowder, R. G. (1974). The modality effect in free and serial recall as a function of phonological similarity. Journal of Verbal Learning \& Verbal Behavior, 13, $430-447$.

Weiskrantz, L. (1968). Memory. In L. Weiskrantz (Ed.), Analysis of behavioral change (pp. 158-188). New York: Harper \& Row.

Weisman, R. G., \& DiFranco, M. P. (1981). Testing models of delayed sequence discrimination in pigeons: Delay intervals and stimulus durations. Journal of Experimental Psychology: Animal Behavior Processes, 7, 413-424.

Weisman, R., Gibson, M., \& Rochford, J. (1984). Testing models of delayed sequence discrimination in pigeons: The advance key procedure. Canadian Journal of Psychology, 38, 256-268.

Weisman, R. G., Wasserman, E. A., Dodd, P. W. D., \& Larew, M. B. (1980). Representation and retention of two-event sequences in pigeons. Journal of Experimental Psychology: Animal Behavior Processes, 6, 312-325.

WINER, B. J. (1971). Statistical principles in experimental design (2nd ed.). New York: McGraw-Hill.

Zentall, T. R., Hogan, D. E., Howard, M. M., \& Moore, B. S. (1978). Delayed matching in the pigeon: Effect on performance of sample-specific observing responses and differential delay behavior. Learning \& Motivation, 9, 202-218.

(Manuscript received May 13, 1985; revision accepted for publication October 18, 1985.) 